\title{
The impact of associated large-fiber peripheral neuropathy on health-related quality of life in Parkinson's disease - results from a Romanian cohort
}

\author{
Oana Maria Vanta', Sebastian Pintea², Lacramioara Perju-Dumbrava ${ }^{3,4}$ \\ ${ }^{1}$ Anatomy Department, "Iuliu Hatieganu" University of Medicine and Pharmacy, Cluj-Napoca, Romania \\ 2Department of Psychology, "Babes-Bolyai" University, Cluj-Napoca, Romania \\ ${ }^{3}$ Neurology I Department, Emergency Clinical County Hospital, Cluj-Napoca, Romania \\ ${ }^{4}$ Neurology Department, "Iuliu Hatieganu" University of Medicine and Pharmacy, \\ Cluj-Napoca, Romania
}

\begin{abstract}
Background. Recent studies described a higher prevalence of peripheral neuropathy (PN) in Parkinson's disease that was linked to L-Dopa exposure. Peripheral neuropathies are known causes of a decreased health-related quality of life (HrQoL). Until now, no studies addressed the issue of how or if associated PN in PD affects HrQoL.

Methods. In a cross-sectional, observational study, 73 non-demented PD patients, from which 36 with confirmed PN based on clinical (using the Toronto Clinical Neuropathy Scale-TCSS) and nerve conduction studies completed the Romanian version of PDQ-39.

Results. Significant differences between mean scores in Motor (49.86 (27.61) vs. 31.50 (26.24), p = 0.005), Activities of daily living (49.86 (27.61) vs. 31.75 (30.10), $p=0.003$ ) and body discomfort (52.54 (29.54) vs. 23.64 (18.48), $p=$ 0.002 ) domains of PDQ-39 in the PN-PD group versus non-PN group were observed. TCSS significantly correlated to motor, emotional well-being and body discomfort domains $(r=0.406 p<0.001 ; r=0.316 p=0.007 ; r=0.356 p=$ 0.002 , respectively). The multivariate linear regression model showed that motor impairment and PN correlated to motor domain (beta $=0.601, p=0.000$; beta $=0.211 p=0.041$, respectively) and PN significantly correlated to body discomfort domain (beta $=0.314, p=0.020$ ) of PDQ-39.

Conclusions. The presence of associated PN in PD determines a further deterioration of $\mathrm{HrQol}$ in subjects with already a poorer $\mathrm{HrQol}$.
\end{abstract}

Keywords: Parkinson's disease, quality of life, large-fiber neuropathy

\section{INTRODUCTION}

Parkinson disease (PD), a prevalent progressive neurodegenerative disorder, causes motor disability as well as non-motor impairment and determines a significant impact on the quality of life (QoL) (1-3).

New data have found a higher prevalence of polyneuropathy in $\mathrm{PD}$ patients than in age-matched controls(4-7). Peripheral nerve fiber impairment in $\mathrm{PD}$ can take the form of large fiber neuropathy $(\mathrm{PN})$ in L-Dopa treated patients (5-12) or small fiber neuropathy (SFN) in all PD patients (13-15). A se- ries of data consider PN to be secondary to L-Dopa intake that determines the accumulation of homocysteine and methylmalonic acid, and cyanocobalamin, pyridoxine or folate plasma deficits (4, $6-8,11,16-18)$. This imbalance in neuro-trophic vitamins and neuro-toxic metabolites is believed to be related to PN in PD (19-21).

Health-related quality of life (HrQoL) in PD is essential in everyday practice because it reflects how a patient perceives the burden of the disease on his daily life and may predict the adherence to treatment or the number of presentation in the clin- 
ic. HrQoL in PD, until recently, was mostly related to the motor impairment of the central nervous system, primarily to motor fluctuations $(2,3,22,23)$, but in the past decades, several papers highlighted that non-motor symptoms in PD determined a worsening in QoL(1,24-26). Psychiatric symptoms are, by far, the non-motor symptoms that have the most significant impact on QoL(26-31), but a recent study showed that the cumulative number of non-motor symptoms in PD is the predictor of a worse Qol in PD(32). Pain in PD is a well known non-motor symptom that can affect QoL $(33,34)$. There are few reports on how peripheral neuropathic pain and sensory neuropathic symptoms influence self-reported QoL in PD $(35,36)$.

Chronic polyneuropathy, regardless of its aetiology, was found to affect all aspects of HrQoL when assessed by the Short Form-36 questionnaire and compared to healthy controls(37). Painful neuropathies were associated with poor $\operatorname{HrQoL}(38,39)$.

Several specific questionnaires, like Parkinson Disease Questionnaire -39 (PDQ-39) and it's Short 8 item form, or Parkinson's Disease Quality of life Questionnaire (PDQL) are recommended for self-assessment of HrQoL in PD(40). Parkinson's Disease Questionnaire 39 and PDQ-39 Summary Index are a validated tool that evaluates HrQol in 8 domains - mobility, activities of daily living, emotional well-being, stigma, social support, cognition, communication and body discomfort (41).

Whatsoever, so far, there are no reports which inquired about QoL in PD patients with associated confirmed PN based, both on clinical and nerve conduction studies criteria. There is a need for reports on how associated PN in PD affects HrQoL in those patients with an already diminished quality of life due to PD itself.

\section{MATERIALS AND METHODS}

This study aimed to evaluate if confirmed PN can affect QoL in PD patients and to what extent.

In an observational, cross-sectional study, we included patients with PD according to UK Parkinson's Disease Brain Bank (42). The subjects were admitted to the Neurology I Department, a general neurology department, of County Emergency Clinical Hospital in Cluj-Napoca from January 2017 to June 2019.
Patients with other known causes of PN, plexopathies, radiculopathies, dementia, or acute medical conditions like stroke, heart attack or infections were excluded.

All patients completed the Romanian version of the PDQ-39 questionnaire in „on” state. The scores for each domain and the PDQ-39 summary Index were calculated accordingly (43). The PDQ- 39 scores range from 0 to 100 , where the higher scores indicate a worse HrQoL.

Relevant data about PD duration, L-Dopa daily dose and psychiatric comorbidities were taken from charts. Neurological examination was made using the UPDRS scale part II - activities of daily living, part III - motor and part IV. Disease severity was assessed on the Hoehn and Yahr scale. Toronto Clinical Neuropathy Scale (TCSS) was used for clinical evaluation of the peripheral nervous system (44). Cognitive functions were evaluated using the universally used Mini-Mental State Examination(MMSE) scale (45).

Al patients underwent electroneurographic evaluation. The full protocol for nerve conduction studies, assessment of the biological parameters and confirmed PN diagnosis, in the cohort were fully detailed in a previous study (46).

All included subjects gave their informed consent. This study was carried under the Helsinki Declaration and received approbation from the local ethics committee.

\section{STATISTICAL ANALYSES}

Statistical analysis was performed using SPSS 20. Descriptive statistics for continuous variables (e.g. age) were presented as mean and standard deviation. Descriptive statistics for categorical variables (e.g. gender) were presented as counts and proportions, and for statistical comparisons of proportions between groups, the Chi-square test was used. Even if the subgroups compared in the first part of the Results section are rather small, the main analysis presented here is parametrical (Independent samples t-tests) since statistical literature proved the robustness of parametrical procedures against non-normality of distributions (47). Anyway, to prove this robustness, the non-parametric version of this analysis (the Mann-Whitney test) is also presented. To overpass the possibility of 
false-positive results due to multiple comparisons, we also analysed the effect size for each comparison (Cohen's d) and decided upon the practical relevance of each result. Correlations between variables were assessed using the Pearson correlation coefficient, followed by multiple linear regression models, in order to have a more comprehensive picture about the predictors of the PDQ-39 domains set. The analysis of scatterplots for the bivariate relationship between each predictor and the criterion variable suggested that a linear analysis was appropriate. For all inferential analysis, a two-sided p-value $<0.05$ was considered statistically significant.

\section{RESULTS}

\section{HrQol in PN versus non-PN groups}

Seventy-three PD patients $(49.3 \%$ females, mean age 65.44 (9.87)) from the previous study were included (46). Two patients scored below 24 points on MMSE but due to motor impairment and 10 patients received medication for anxiety or depression. Those patients were still included. The entire group was separated into 2 subgroups - PN and non-PN. The PN group included 36 subjects with associated confirmed PN, evaluated in the previous study. The non-PN group included 37 subjects without PN. The PN group differed significantly from the non-PN regarding disease duration $(p<0.001)$, age $(\boldsymbol{p}=0.051)$, L-Dopa daily dose ( $p$ 0.023), MMSE score ( $p=0.003$ and UPDRS activities of daily living (ADL) score, motor and part IV $\operatorname{scores}(p=0.003, \mathrm{p}=0.007, \mathrm{p}=0.007$, respec- tively). The non-parametric analysis confirmed the parametric results. Effect sizes varied from moderate to large. Demographic and clinical characteristics of the 2 groups are summarized in Table 1.

PN patients experienced a significant worse QoL compared to non-PNP in all domains, except stigma and social support (34.43 (30.79) vs. 25.34 (26.43), $p=0.180 ; 20.54$ (26.38) vs. 14.30 (19.42), $p=0.253)$, respectively. Significant differences between mean scores in motor, ADL and body discomfort domains of PDQ 39 in the 2 groups were observed. The non-parametric analysis confirmed the parametric results. Effect sizes varied from small (the case of non-significant differences) to moderate and large (the case of significant differences) (Table 2).

TCSS score positively correlated to motor domain ( $r=0.406, p<0.001)$, activities of daily living $(r=0.325, p<0.05)$ and total score $(r=0.416$, $p<0.001)$. Body discomfort was influenced only by TCSS score $(r=0.356, p<0.01)$, and UPDRS part IV-motor complications score $(r=0.255, p<0.05)$. Age did not influence any domains of the PDQ-39 questionnaire. Significant correlations were observed between UPDRS scores, MMSE and PDQ39 domains (Table 3 ).

\section{Multivariate linear regression analyses}

A multivariate linear regression model with the PDQ-39 domains and PDQ39-SI set as dependent variables and age, TCSS, UPDRS Motor score (part III) and MMSE as predictors was run. After adjusting for those variables, $\mathrm{PN}$ - as assessed by TCSS, still significantly correlated to the body dis-

TABLE 1. Demographic and clinical characteristics of PD patients with and without peripheral neuropathy (Independent sample t-Test/ Chi-square test, Mann-Whitney test and Cohen's d). Results are represented as mean (SD). F- female, $M-$ male, LDD - L-dopa daily dose

\begin{tabular}{|l|c|c|c|c|c|}
\hline & $\begin{array}{c}\text { PN group } \\
\mathbf{N = 3 6}\end{array}$ & $\begin{array}{c}\text { Non-PN group } \\
\mathbf{N = 3 7}\end{array}$ & $\boldsymbol{p}$ (t-test) & $\boldsymbol{p}$ (M-W test) & Cohen's $\boldsymbol{d}$ \\
\hline Age (years) & $67.72(8.83)$ & $63.22(10,43)$ & 0.051 & 0.072 & 0.46 \\
\hline Sex (F:M) & $15: 21$ & $21: 16$ & 0.245 & - & - \\
\hline Hoehn and Yahr stage & $3.28(0.67)$ & $2.46(0.83)$ & 0.000 & 0.001 & 1.08 \\
\hline PD Duration (years) & $9.18(5.049)$ & $4.54(4.78)$ & 0.000 & 0.001 & 0.94 \\
\hline LDD (mg/day) & $984.23(441.94)$ & $702.06(317.14)$ & 0.023 & 0.020 & 0.73 \\
\hline UPDRS ADL score & $15.81(8.73)$ & $9.68(8.27)$ & 0.003 & 0.001 & 0.72 \\
\hline UPDRS MOTOR Score & $21.64(9.71)$ & $15.35(9.69)$ & 0.007 & 0.001 & 0.64 \\
\hline UPDRS part IV & $5.33(5.16)$ & $2.49(3.35)$ & 0.007 & 0.007 & 0.65 \\
\hline MMSE & $26.67(2.02)$ & $27.97(1.62$ & 0.003 & 0.006 & 0.71 \\
\hline TCSS & $10.31(4.86)$ & $3.81(3.46)$ & 0.000 & 0.001 & 1.54 \\
\hline
\end{tabular}


TABLE 2. Domain scores of PDQ-39 and PDQ39-SI in the PD patients with and without peripheral neuropathy. (Independent sample t-Test, Mann-Whitney test and Cohen's d). Results are represented as mean (SD)

\begin{tabular}{|l|c|c|c|c|c|}
\hline PDQ Domains scores & $\begin{array}{c}\mathbf{P N} \\
\mathbf{( N = 3 6 )}\end{array}$ & $\begin{array}{c}\text { NonPNP } \\
\mathbf{( N = 3 7 )}\end{array}$ & $\boldsymbol{p}$ (t-test) & $\boldsymbol{p}$ (M-W test) & Cohen's $\boldsymbol{d}$ \\
\hline MOTOR & $49.86(27.61)$ & $31.50(26.24)$ & 0.005 & 0.005 & 0.68 \\
\hline ADL & $52.88(28.43)$ & $31.75(30.10)$ & 0.003 & 0.003 & 0.72 \\
\hline EMOTIONS & $43.51(24.47)$ & $28.93(23.49)$ & 0.011 & 0.012 & 0.60 \\
\hline STIGMA & $34.43(30.79)$ & $25.34(26.43)$ & 0.180 & 0.191 & 0.31 \\
\hline SOCIAL SUPORT & $20.54(26.38)$ & $14.30(19.42)$ & 0.253 & 0.368 & 0.27 \\
\hline COGNITION & $41.63(21.53)$ & $22.47(20.17)$ & 0.001 & 0.001 & 0.91 \\
\hline COMUNICATION & $36.79(26.46)$ & $23.64(18.48)$ & 0.016 & 0.038 & 0.57 \\
\hline BODY DISCOMFORT & $52.54(29.54)$ & $33.10(22.26)$ & 0.002 & 0.003 & 0.74 \\
\hline SUMMARY INDEX & $42.81(19.59)$ & $26.85(16.43)$ & 0.001 & 0.001 & 0.88 \\
\hline
\end{tabular}

TABLE 3. Correlations between demographic and clinical parameters and PDQ-39 domains and PDQ39-SI (r-Pearson correlation coefficient). Results of particular interest are in Bold

\begin{tabular}{|c|c|c|c|c|c|c|c|c|c|c|}
\hline & & MOTOR & $A D L$ & EMOTIONS & STIGMA & $\begin{array}{c}\text { SOCIAL } \\
\text { SUPORT }\end{array}$ & COGNITION & COMUNICATION & $\begin{array}{c}\text { BODY } \\
\text { DISCOMFORT }\end{array}$ & PDQ SI \\
\hline \multirow{2}{*}{ Age } & $r$ & 0.176 & 0.153 & 0.059 & 0.083 & -0.115 & 0.187 & 0.121 & 0.160 & 0.157 \\
\hline & $p$ & 0.137 & 0.196 & 0,619 & 0.485 & 0,332 & 0.113 & 0.309 & 0.176 & 0.183 \\
\hline \multirow{2}{*}{ TCSS } & $r$ & 0.406 & 0.325 & 0.316 & 0.166 & 0.019 & 0.413 & 0.263 & 0.356 & 0.416 \\
\hline & $p$ & 0.000 & 0.005 & 0.007 & 0.160 & 0.875 & 0.000 & 0.024 & 0.002 & 0.000 \\
\hline \multirow{2}{*}{$\begin{array}{l}\text { UPDRS } \\
\text { ADL } \\
\text { SCORE } \\
\end{array}$} & $r$ & 0.682 & 0.708 & 0.374 & 0.115 & 0.018 & 0.487 & 0.470 & 0.198 & 0.532 \\
\hline & $p$ & 0.000 & 0.000 & 0.001 & 0.333 & 0.883 & 0.000 & 0.000 & 0.093 & 0.000 \\
\hline \multirow{2}{*}{$\begin{array}{l}\text { UPDRS } \\
\text { MOTOR } \\
\text { SCORE }\end{array}$} & $r$ & 0.670 & 0.710 & 0.423 & 0.189 & 0.159 & 0.437 & 0.497 & 0.214 & 0.572 \\
\hline & $p$ & 0.000 & 0.000 & 0.000 & 0.109 & 0.180 & 0.000 & 0.000 & 0.069 & 0.000 \\
\hline \multirow{2}{*}{$\begin{array}{l}\text { UPDRS } \\
\text { PART IV } \\
\text { SCORE }\end{array}$} & $r$ & 0.510 & 0.539 & 0.395 & 0.179 & 0.195 & 0.433 & 0.445 & 0.255 & 0.504 \\
\hline & $p$ & 0.000 & 0.000 & 0.001 & 0.130 & 0.098 & 0.000 & 0.000 & 0.030 & 0.000 \\
\hline \multirow{2}{*}{ MMSE } & $r$ & 0.336 & 0.438 & 0.219 & 0.203 & 0.183 & 0.352 & 0.276 & 0.164 & 0.379 \\
\hline & $p$ & 0.004 & 0.000 & 0.063 & 0.084 & 0.121 & 0.002 & 0.018 & 0.166 & 0.001 \\
\hline
\end{tabular}

comfort domain (beta $=0.314, p=0.020)$ of PDQ -39. UPDRS part III and PN were found to have a more significant impact on overall HrQoL as evaluated by PDQ39 - SI $($ beta $=0.451, p=0.000$; beta $=$ $0,253, p=0.025$, respectively) and on motor domain of PDQ-39 $($ beta $=0.601, p=0.000 ;$ beta $=0.211 p$ $=0.041$, respectively). Interpretation of Beta coefficients (as standardised correlation coefficients) was according to Cohen (1988), with values above 0.50 considered large, around 0.30 considered moderate and values around 0.10 interpreted as small effects (48). Results are summarised in Table 4.

\section{DISCUSSION}

Patients in the PN group were older, in more advanced stages, with longer PD duration and scored higher in the UPDRS scale, than those in the non-
PN group. PN in this cohort was previously shown to be related to L-Dopa intake; possible causes and relations of PN to PD in this cohort were previously discussed and PN was linked to vitamin B12 and folate deficiencies and high daily doses of L-Dopa (46). Nevertheless, PN in PD seems to be more common in advanced stages of PD and linked to higher doses of L-Dopa and might represent a more severe phenotype of PD (49).

This study included patients with confirmed PN of no other causes, excluding patients with plexopathy or radiculopathy; thus, these results depict the impact of signs and symptoms of PN on HrQoL, rather than focusing on specific sensory symptoms or neuropathic pain that are already predictors for a poor HrQoL $(35,36)$. To the best of our knowledge, this is the first study to evaluate HrQol of PD patients with associated large fiber neuropathy. 
TABLE 4. Results of multiple linear regression models: effect of PN on PDQ-39 domains and PDQ-SI. Outcomes of particular interest are in Bold and Italics

\begin{tabular}{|l|c|c|c|c|c|c|c|c|c|c|}
\hline & & MOTOR & ADL & EMOTION & STIGMA & $\begin{array}{c}\text { SOCIAL } \\
\text { SUPORT }\end{array}$ & COGNITION & COMUNICATION & $\begin{array}{c}\text { BODY } \\
\text { DISCOMFORT }\end{array}$ & $\begin{array}{c}\text { PDQ 39 } \\
\text { SI }\end{array}$ \\
\hline \multirow{2}{*}{ TCSS } & Beta & $\mathbf{0 . 2 1 1}$ & 0.079 & 0.239 & 0.090 & 0.000 & $\mathbf{0 . 2 2 7}$ & 0.104 & $\mathbf{0 . 3 1 4}$ & $\mathbf{0 . 2 5 3}$ \\
& $p$ & $\mathbf{0 . 0 4 1}$ & 0.420 & 0.060 & 0.514 & 0.999 & $\mathbf{0 . 0 2 4}$ & 0.398 & $\mathbf{0 . 0 2 0}$ & $\mathbf{0 . 0 2 5}$ \\
\hline \multirow{2}{*}{ Age } & Beta & 0.019 & 0.010 & -0.085 & -0.004 & 0.181 & -0.002 & 0.014 & 0.012 & -0.032 \\
& $p$ & 0.849 & 0.915 & 0.481 & 0.976 & 0.174 & 0.987 & 0.907 & 0.925 & 0.765 \\
\hline \multirow{2}{*}{ UPDRS } & Beta & $\mathbf{0 . 6 0 1}$ & 0.624 & 0.354 & 0.108 & 0.097 & $\mathbf{0 . 2 9 7}$ & 0.443 & 0.115 & $\mathbf{0 . 4 5 1}$ \\
MOTOR & $p$ & $\mathbf{0 . 0 0 0}$ & 0.000 & 0.005 & 0.415 & 0.464 & $\mathbf{0 . 0 1 2}$ & 0.000 & 0.369 & $\mathbf{0 . 0 0 0}$ \\
\hline \multirow{2}{*}{ MMSE } & Beta & -0.007 & -0.148 & -0.013 & -0.128 & -0.194 & -0.134 & -0.052 & -0.005 & -0.113 \\
& $p$ & 0.943 & 0.127 & 0.916 & 0.348 & 0.155 & 0.262 & 0.669 & 0.971 & 0.304 \\
\hline
\end{tabular}

PDQ-39 scores and PDQ-39-SI were significantly higher in the PN group than in the non-PN group, demonstrating that subjects in the PN group experienced a worse HrQoL compared to non-PNP in all domains, except stigma and social support.

These results highlighted that the associated impairment of peripheral nervous system had a significant impact on HrQoL in PD patients in the motor, activities of daily living, emotional well-being and body discomfort domains of HrQoL, as well as overall.

Motor disability, as assessed by UPDRS motor score showed significant correlations to the motor domain and activities of daily living of PDQ-39, as expected because, until recently, HrQoL in PD related significantly to motor impairment $(2,3)$. After adjusting for this score, the multivariate regression model showed that polyneuropathy contributes to the burden of motor disability in everyday life, and may determine a further deterioration of HrQoL. Taken together, motor impairment and polyneuropathy had a substantial impact on overall HrQoL in $\mathrm{PD}$, as shown by the multivariate regression model.

Motor complications, as assessed by UPDRS score part IV, influenced overall HrQoL and subdomains like motor function, activities of daily living or communication. Similar results were already outlined in other studies $(22,23)$.

The multivariate regression model showed that the body discomfort domain was only influenced by PN, suggesting that positive sensory symptoms, like paresthesia, are responsible for poorer $\mathrm{HrQoL}$ in this aspect.

Stigma and social support domains were not influenced by motor impairment, cognitive functions as assessed by MMSE, or polyneuropathy when taken separately nor in the multivariate regression model. These results indicate that these specific domains- stigma and social support can be instead more related to an individual's personality features, education levels and family dynamics.

Caution should be taken when interpreting these results, because this study was cross-sectional, on small sample size and single centre-based. Because of the study design, these associations cannot define a causal relationship. Furthermore, we did not assess the impact of non-motor symptoms, nor did we adjust the results for the presence of anxiety or depression. Non-motor symptoms, especially neuropsychiatric ones, are known predictors for worse HrQoL in PD (26).

The modality in which a patient experiences the burden of a chronic disease like PD is individual and is multifactorial influenced, as these results also show. The association of PN in PD may determine a further deterioration of HrQoL in subjects with already a poorer $\mathrm{HrQoL}$ as these results also show.

So far, there are no clinical good practice recommendations for treating a PD patient with $\mathrm{PN}$, nor how to evaluate PD-associated PN. We believe these issues should be addressed in further studies, and guidelines are needed to be available, for patients to benefit from high-quality medical care.

\section{CONCLUSIONS}

Large fiber neuropathy was found to be more common in the advanced stages of Parkinson's disease. The impact of an associated condition like peripheral neuropathy caused a further motor impairment, as perceived by the subjects, which had a negative influence on health-related quality of life in PD patients. Patients with large fiber neuropathy and PD experienced worse quality of life as PD pa- 
tients without PN. Peripheral neuropathy and motor disability were the predictors for a poorer HrQoL in this cohort of PD patients from Transilvania, Romania.

There is a need for large-scale, prospective studies to evaluate how exactly large fiber impairment progresses in PD and how it impacts HrQoL when adjusting for non-motor symptoms, or after treatment of PN.

\section{REFERENCES}

1. Barone P, Antonini A, Colosimo C, Marconi R, Morgante L, Avarello TP, et al. The PRIAMO study: A multicenter assessment of nonmotor symptoms and their impact on quality of life in Parkinson's disease. Movement Disorders 2009; 24(11):1641-9.

2. Dodel RC, Berger $\mathrm{K}$, Oertel WH. Health-related quality of life and healthcare utilisation in patients with Parkinson's disease: Impact of motor fluctuations and dyskinesias. PharmacoEconomics. 2001; 19(10):1013-38.

3. Hechtner MC, Vogt T, Zöllner Y, Schröder S, Sauer JB, Binder H, et al. Quality of life in Parkinson's disease patients with motor fluctuations and dyskinesias in five European countries. Parkinsonism and Related Disorders. 2014;20(9):969-74.

4. Rajabally YA, Martey J. Neuropathy in Parkinson disease Prevalence and determinants. Neurology. 2011;77(22):1947-50.

5. Szadejko K, Dziewiatowski K, Szabat K, Robowski P, Schinwelski M, Sitek E, et al. Polyneuropathy in levodopa-treated Parkinson's patients. Journal of the Neurological Sciences. 2016;371:36-41.

6. Toth C, Breithaupt K, Ge S, Duan Y, Terris JM, Thiessen A, et al. Levodopa, methylmalonic acid, and neuropathy in idiopathic Parkinson disease. Annals of Neurology. 2010;68(1):28-36.

7. Toth C, Brown MS, Furtado S, Suchowersky O, Zochodne D. Neuropathy as a potential complication of levodopa use in Parkinson's disease. Movement Disorders. 2008;23(13):1850-9.

8. Ceravolo R, Cossu G, Bandettini di Poggio M, Santoro L, Barone P, Zibetti M, et al. Neuropathy and levodopa in Parkinson's disease: Evidence from a multicenter study. Movement Disorders. 2013; 28(10):1391-7.

9. Grambalova Z, Kaiserova M, Vastik M, Mensíkova K, Otruba P, Zapletalova J, Dufek J, Kanovsky P. Peripheral neuropathy in Parkinson's disease. Neuro Endocrinol Lett. 2015;36(4):363-7.

10. Jugel C, Ehlen F, Taskin B, Marzinzik F, Müller T, Klostermann F. Neuropathy in Parkinson's Disease Patients with Intestinal Levodopa Infusion versus Oral Drugs. PLOS ONE. 2013;8(6):1-6.

11. Loens S, Chorbadzhieva E, Kleimann A, Dressler D, Schrader C. Effects of levodopa/carbidopa intestinal gel versus oral levodopa/ carbidopa on B vitamin levels and neuropathy. Brain and Behavior. 2017;7(5):1-8.

12. Cossu G, Ceravolo R, Zibetti M, Arca R, Ricchi V, Paribello A, et al. Levodopa and neuropathy risk in patients with Parkinson disease: Effect of COMT inhibition. Parkinsonism and Related Disorders. 2016;27(September 2014):81-4.

13. Doppler K, Ebert S, Üçeyler N, Trenkwalder C, Ebentheuer J, Volkmann J, et al. Cutaneous neuropathy in Parkinson's disease: A window into brain pathology. Acta Neuropathologica. 2014;128(1):99109.

14. Kass-lliyya L, Javed S, Gosal D, Kobylecki C, Marshall A, Petropoulos IN, et al. Small fiber neuropathy in Parkinson's disease: A clinical, pathological and corneal confocal microscopy study. Parkinsonism and Related Disorders. 2015;21(12):1454-60.

15. Nolano M, Provitera V, Manganelli F, lodice R, Stancanelli A, Caporaso G, et al. Loss of cutaneous large and small fibers in naive and I -dopa-treated PD patients. Neurology. 2017;89(8):776-84.

16. Andréasson M, Brodin L, Laffita-Mesa JM, Svenningsson P. Correlations between Methionine Cycle Metabolism, COMT Genotype, and Polyneuropathy in L-Dopa Treated Parkinson's
Disease: A Preliminary Cross-Sectional Study. Journal of Parkinson's Disease. 2017;7(4):619-28.

17. Merola A, Zibetti M, Rizzone MG, Troiano M, Artusi CA, Angrisano S, et al. Prospective assessment of peripheral neuropathy in Duodopatreated parkinsonian patients. Acta Neurologica Scandinavica. 2014;129(1):1-5.

18. Rajabally YA, Martey J. Levodopa, vitamins, ageing and the neuropathy of Parkinson's disease. Journal of Neurology. 2013; 260(11):2844-8.

19. Müller T, Laar TV, Cornblath DR, Odin P, Klostermann F, Grandas FJ, et al. Peripheral neuropathy in Parkinson's disease: Levodopa exposure and implications for duodenal delivery. Parkinsonism and Related Disorders. 2013;19(5):501-7.

20. Romagnolo A, Merola A, Artusi CA, Rizzone MG, Zibetti M, Lopiano L. Levodopa-Induced Neuropathy: A Systematic Review. Movement Disorders Clinical Practice. 2019;6(2):96-103.

21. Cossu G, Melis M. The peripheral nerve involvement in Parkinson Disease: A multifaceted phenomenon. Parkinsonism and Related Disorders. 2016;25:17-20.

22. Onozawa R, Tsugawa J, Tsuboi Y, Fukae J, Mishima T, Fujioka S. The impact of early morning off in Parkinson's disease on patient quality of life and caregiver burden. Journal of the Neurological Sciences. 2016;364:1-5.

23. Perez-Lloret S, Negre-Pages L, Damier P, Delval A, Derkinderen $P$, Destée A, et al. L-DOPA-induced dyskinesias, motor fluctuations and health-related quality of life: the COPARK survey. European Journal of Neurology. 2017;24(12):1532-8.

24. Barone P, Erro R, Picillo M. Quality of Life and Nonmotor Symptoms in Parkinson's Disease. International Review of Neurobiology. 133: Elsevier; 2017. p. 499-516.

25. Martinez-Martin P, Rodriguez-Blazquez C, Kurtis MM, Chaudhuri KR. The impact of non-motor symptoms on health-related quality of life of patients with Parkinson's disease. Movement Disorders. 2011; 26(3):399-406.

26. Balestrino R, Martinez-Martin P. Neuropsychiatric symptoms, behavioural disorders, and quality of life in Parkinson's disease. Journal of the Neurological Sciences. 2017;373:173-8.

27. Lawson RA, Yarnall AJ, Duncan GW, Khoo TK, Breen DP, Barker RA, et al. Quality of life and mild cognitive impairment in early Parkinson's disease: does subtype matter? Journal of Parkinson's disease. 2014;4(3):331-6.

28. Marras C, McDermott MP, Rochon PA, Tanner CM, Naglie G, Lang $A E$, et al. Predictors of deterioration in health-related quality of life in Parkinson's disease: results from the DATATOP trial. Movement Disorders 2008; 23(5):653-9; quiz 776.

29. Nicoletti A, Mostile G, Stocchi F, Abbruzzese G, Ceravolo R, Cortelli $P$, et al. Factors influencing psychological well-being in patients with Parkinson's disease. PLoS ONE. 2017;12(12):1-8.

30. Santos-García D, de la Fuente-Fernández R. Impact of non-motor symptoms on health-related and perceived quality of life in Parkinson's disease. Journal of the Neurological Sciences. 2013; 332(1-2):136-40.

31. Pontone GM, Bakker CC, Chen S, Mari Z, Marsh L, Rabins PV, et al. The longitudinal impact of depression on disability in Parkinson disease. International Journal of Geriatric Psychiatry. 2016; 31(5):458-65. 
32. Duncan GW, Khoo TK, Yarnall AJ, O'Brien JT, Coleman SY, Brooks DJ, et al. Health-related quality of life in early Parkinson's disease: The impact of nonmotor symptoms. Movement Disorders. 2014; 29(2):195-202.

33. Choi SM, Kim BC, Jung HJ, Yoon GJ, Kang KW, Choi KH, et al. Impact of pain and pain subtypes on the quality of life of patients with Parkinson's disease. Journal of Clinical Neuroscience. 2017; 45:105-9.

34. Martinez-Martin P, Manuel Rojo-Abuin J, Rizos A, RodriguezBlazquez C, Trenkwalder C, Perkins $L$, et al. Distribution and impact on quality of life of the pain modalities assessed by the King's Parkinson's disease pain scale. npj Parkinson's Disease. 2017; 3(1):1-5.

35. Adewusi JK, Hadjivassiliou M, Vinagre-Aragón A, O'Connor KR, Khan A, Grünewald RA, et al. Sensory neuropathic symptoms in idiopathic Parkinson's disease: prevalence and impact on quality of life. Acta Neurologica Belgica. 2018;118(3):445-50.

36. Adewusi JK, Hadjivassiliou M, Vinagre-Aragón A, O'Connor KR, Khan A, Grünewald RA, et al. Peripheral neuropathic pain in idiopathic Parkinson's disease: Prevalence and impact on quality of life; a case controlled study. Journal of the Neurological Sciences. 2018;392(March):3-7.

37. Panduranga MS, Vibha D, Prasad K, Srivastava AK, Shukla G. Clinical spectrum and quality of life in patients with chronic polyneuropathy: A cross-sectional study. Journal of the Peripheral Nervous System. 2018;23(2):120-3.

38. Girach A, Julian TH, Varrassi G, Paladini A, Vadalouka A, Zis P. Quality of life in painful peripheral neuropathies: A systematic review. Pain Research and Management. 2019;2019.

39. Zis P, Sarrigiannis PG, Rao DG, Hewamadduma C, Hadjivassiliou M. Chronic idiopathic axonal polyneuropathy: Prevalence of pain and impact on quality of life. Brain and Behavior. 2019;9(1):1-6.

40. Martinez-Martin P, Jeukens-Visser M, Lyons KE, Rodriguez-Blazquez C, Selai C, Siderowf A, et al. Health-related quality-of-life scales in
Parkinson's disease: Critique and recommendations. Movement Disorders. 2011;26(13):2371-80.

41. Jenkinson C, Fitzpatrick R, Peto V, Greenhall R, Hyman N. The Parkinson's Disease Questionnaire (PDQ-39): development and validation of a Parkinson's disease summary index score. Age and ageing. 1997;26(5):353-7.

42. Hughes AJ, Daniel SE, Kilford L, Lees AJ. Accuracy of clinical diagnosis of idiopathic Parkinson's disease: A clinico-pathological study of 100 cases. Journal of Neurology Neurosurgery and Psychiatry. 1992;55(3):181-4.

43. Souza RG, Borges V, Silva SM, Ferraz HB. Quality of life scale in Parkinson's disease PDQ-39 - (Brazilian Portuguese version) to assess patients with and without levodopa motor fluctuation. Arq Neuropsiquiatr. 2007;65(3B):787-91.

44. Bril V, Perkins BA. Validation of the Toronto clinical scoring system for diabetic polyneuropathy. Diabetes Care. 2002;25(11):2048-52.

45. Folstein MF, Folstein SE, McHugh PR. "Mini-mental state". A practical method for grading the cognitive state of patients for the clinician. J Psychiatr Res. 1975;12(3):189-98.

46. Vanta OM, Tohanean N. Pintea S.; Perju-Dumbrava, L. Large-Fiber Neuropathy in Parkinson's Disease: Clinical, Biological, and Electroneurographic Assessment of a Romanian Cohort. Journal of Clinical Medine. 2019;8.

47. Rasch DG, Volker. The robustness of parametric statistical methods. Psychology Science. 2004;46(2):175-208.

48. Cohen JA. Statistical Power Analysis for the Behavioral Sciences. second edition ed: Lawrence Erlbaum associetes, Publishers; 1988. $567 \mathrm{p}$.

49. Merola A, Rosso M, Romagnolo A, Comi C, Fasano A, Zibetti M, et al. Peripheral neuropathy as marker of severe Parkinson's disease phenotype. Movement Disorders. 2017;32(8):1256-8. 